\title{
Review Paper: Role of Nitric Oxide on Dopamine Release and Morphine-Dependency
}

\author{
Amir Arash Motahari' ${ }^{1}$, Hedayat Sahraei ${ }^{1}$, Gholam Hossein Meftahi ${ }^{1^{*}}$
}

1. Neuroscience Research Center, Baqiyatallah University of Medical Sciences, Tehran, Iran.

ditation: Motahari, A. A., Sahraei, H., \& Meftahi, Gh. H. (2016). Role of nitric oxide on dopamine release and morphine-dependency. Basic and Clinical Neuroscience, 7(4), 283-290. http://dx.crossref.org/10.15412/J.BCN.03070401

http://dx.crossref.org/10.15412/J.BCN.03070401

Article info:

Received: 07 April 2016

First Revision: 04 May 2016

Accepted: 01 September 2016

Key Words:

Nitric oxide, Opioid,

Amygdala, Ventral

tegmental area, Nucleus

accumbens

\begin{abstract}
A B S T RA C T
The catastrophic effects of opioids use on public health and the economy are documented clearly in numerous studies. Repeated morphine administration can lead to either a decrease (tolerance) or an increase (sensitization) in its behavioral and rewarding effects. Morphine-induced sensitization is a major problem and plays an important role in abuse of the opioid drugs. Studies reported that morphine may exert its effects by the release of nitric oxide (NO). NO is a potent neuromodulator, which is produced by nitric oxide synthase (NOS). However, the exact role of $\mathrm{NO}$ in the opioid-induced sensitization is unknown. In this study, we reviewed the role of NO on opioid-induced sensitization in 2 important, rewarding regions of the brain: nucleus accumbens and ventral tegmentum. In addition, we focused on the contribution of $\mathrm{NO}$ on opioid-induced sensitization in the limbic system.
\end{abstract}

\section{Introduction}

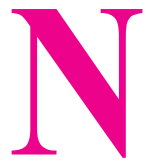

itric oxide (NO) is synthesized by nitric oxide synthase (NOS) enzyme from Larginine in many mammalian cells. NO is a potent stimulator of guanylate cyclase (GC), resulting in increased levels of cyclic guanosine monophosphate (cGMP) (Bahari, et al., 2014). It has a critical role in a variety of physiological and pathophysiological functions, such as opioidsinduced sensitization. High prevalence of opiate addiction in many countries, including Iran has created a lot of concerns and problems. Lack of appropriate treatment for drug dependency is partially due to unknown effects of morphine on different areas of the nervous system. Thus, the investigation of drug dependency is under consideration.
It is well-known that long-term use of opioids can lead to tolerance to their effects. Tolerance results primarily from adaptive neuronal changes due to long-term use of opioids, which gradually decreases the effects of opioids (Erfani, Sahraei, \& Meftahi, 2015; Yamada, 2008). Tolerance to the rewarding effects of opioids, which is called motivational tolerance, is the main reason for the increased use of these drugs. The potential factor(s) that generated opioids tolerance is unknown. However, recent studies showed that NO has an important role in the opioids tolerance and targeting NOS may provide a novel treatment avenue. In this review, we will discuss many pharmacological studies about the role of NO in the opioids-induced sensitization. We will focus on the role of NO in some important regions of the brain that involved in opioids-induced sensitization, such as the

* Corresponding Author:

Gholam Hossein Meftahi, PhD

Address: Neuroscience Research Center, Baqiyatallah University of Medical Sciences, Araj St., Niavaran, Tehran, Iran. PO Box: 19395-6558

Tel: +98 (21) 26127286

E-mail:meftahi208@yahoo.com 
nucleus accumbens (NA), the ventral tegmental area (VTA), and the limbic system.

\section{Role of Nitric Oxide in Morphine-Induced Sensitization}

Morphine-induced sensitization is a major problem and plays an important role in abuse liability of the opioid drugs (Robinson and Berridge, 2003). Dopamine (DA) and glutamate (Di Chiara, 2002; Siggins et al., 2003) pathways are involved during morphine-induced sensitization. Several studies indicate that NO plays an important role in morphine-induced sensitization in rodents (Sahraei et al., 2007; Zarrindast, Gholami, Sahraei, \& Haeri-Rohani, 2003).

Morphine-induced sensitization is accompanied with a rise in the ability of opioids to promote DA release in NA and VTA brain regions that are thought to be involved in this process (Yaribeygi et al., 2014). NA is a complex forebrain structure, involved in the regulation of motivation and motor behavior. Some evidence suggests that morphine-induced sensitization causes many longlasting changes in NA neurons. For example, dopamine D1 receptors in NA become hypersensitive after sensitization, presumably further potentiating the mesolimbic dopamine signal.

In addition to the dopaminergic pathways, the involvement of non-dopaminergic neurotransmitter and neuromodulatory systems in morphine-induced sensitization of NA have been recently documented. Recently, studies suggest that $\mathrm{NO}$ is the most prominent signaling molecule that is involved in morphine-induced sensitization. Some studies showed the high concentration of NOS in NA region.

Moreover, nitric oxide can interact with dopamine and glutamate systems in several brain areas such as NA (Sahraei et al., 2007; Zarrindast et al., 2003). Sahraei et al. showed that intra-NA infusion of L-arginine (NO precursor) would inhibit both the acquisition and expression of morphine-induced conditioned place preference (CPP) in rats (2007). However, co-administration of morphine and L-arginine decreases the acquisition of morphine-induced CPP and behavioral sensitization (Sahraei et al., 2004a; Sahraei et al., 2007). The nature of this phenomenon is not well understood.

The controversy seems to arise from the differences in the genetic background, environment, and methods used in these studies. L-arginine increases NO level in several brain regions (Wiesinger, 2001; Prast and Philippu,
2001) and it is a powerful mediator for inhibiting DA transporters, which take-up DA (Kiss, 2000; Kiss \& Vizi, 2001; Wiesinger, 2001). Hence, any increase in NO level by L-arginine in NA may decrease DA reuptake, thereby raises the concentration of synaptic DA, which may account for the L-arginine effects on morphine CPP (both in its acquisition and expression).

Moreover, L-arginine by itself can release DA (Wiesinger, 2001) which may also account for the L-arginine response. A wealth of evidence indicates that an increase in NO concentration could change the function of other neurotransmitters such as serotonin, glutamate, GABA, and acetylcholine (ACh) (Prast, Tran, Fischer, \& Philippu, 1998; Trabace et al., 2004). Therefore, infusion of L-arginine in NA can lead to an increase in NO generation, which in fact changes its concentration and function of several neurotransmitters, including DA, glutamate, GABA, and serotonin. For example, glutamate exerts its effects by activation of several mechanisms, including an increase in NOS activity (Ohno, Arai, \& Watanabe, 1995).

An interesting finding showed that intra-NA administration of L-arginine in the control animals increases their response to an ineffective dose of morphine i.e., Larginine induces CPP in control animals (Sahraei, et al., 2007; Sahraei et al., 2004a; Zarrindast, Karami, Sepehri, $\&$ Sahraei, 2002). Considering the role of NO on morphine-induced sensitization in NA, L-arginine might interact with DA or glutamate in NA for induction of sensitization and cross-sensitization to morphine. However, the exact mechanism of this phenomenon must be further studied.

In our laboratory, intra-NA administration of L-NAME (L-arginine methyl ester) (a NOS inhibitor) also inhibited both the acquisition and expression of morphine CPP in rats. However, some studies showed that application of L-NAME produces no effect on morphine-induced CPP (Sahraei et al., 2007; Sahraei et al., 2004a). So, we postulate that an increase or decrease in NO concentration in NA leads to an imbalance between the function of several neurotransmitter pathways and disrupts the concert harmony between these pathways.

\section{Role of Nitric Oxide in Dopamine Release in the Nucleus Accumbens}

Nucleus accumbens, the major part of the limbic ventral striatum, has an important role in drug addiction. Afferents from limbic-associated brain regions such as medial prefrontal cortex, basolateral amygdala, and ventral subiculum of the hippocampus converge in NA 
(French \& Totterdell, 2002; Hosseini et al., 2015; Sesack $\&$ Grace, 2010). These afferents integrate with thalamic input and interface with motor loops of the basal ganglia (Groenewegen \& Trimble, 2007; Sesack \& Grace, 2010; Zahm, 2000). These interactions are controlled by dopaminergic pathways from VTA (Ikemoto, 2007).

Some studies revealed that the firing rates of the dopaminergic neurons are changed by electrical stimulations (Matsumoto \& Hikosaka, 2009; Schultz, 2002), and these changes can influence the firing of action potentials in NA (Morris et al., 2010; Schultz, 2010). DA release in NA is monitored by several signaling pathways (Cragg, 2006). NO is a pivotal neuromodulator that is thought to be produced locally in NA interneurons by NOS (French, Ritson, Hidaka, \& Totterdell, 2005; Kraus \& Prast, 2001). However, the mechanism(s) of NO action in signaling of DA in NA is (are) unknown.

Hartung et al. showed that NO donors increased DA release evoked by a range of different electrical stimulations (with a frequency of 1 to $100 \mathrm{~Hz}$ ) in NA. They also showed that NO donors enhanced DA-evoked release in NA with increasing stimulus frequency (Hartung, Threlfell, \& Cragg, 2011). Also, these NO-mediated effects were independent of sGC (soluble guanylyl cyclase) activation, DA transporters, and BK channels (large conductance $\mathrm{Ca}^{2 \pm}$ activated $\mathrm{K}^{+}$channels). Furthermore, this frequency-dependent modulation of DA release by NO can be modulated by $\mathrm{ACh}$. Hartung et al. also revealed that in NA, interactions between NO, ACh, and DA may be important for regulating these behaviors in normal and pathological states. The physiological concentrations of NO are still debated (Hall \& Garthwaite, 2009).

It seems that $\mathrm{NO}$ concentration in normal tissues ranges from hundreds of picomolar to low-nanomolar (Hall \& Garthwaite, 2009). Many target molecules are identified in modulating the physiological functions of NO. Most probably, sGC enzyme is an important mechanism for some actions of NO. However, Stewart et al. showed the sGC-independent effects of NO on DA release in the dorsal striatum (Stewart et al, 1996). Some studies suggested that NO increases extracellular striatal DA levels by inhibition of DA transporters (Lonart \& Johnson, 1994).

The conductance of BK channels (among other $\mathrm{K}^{+}$channels) is also reported to be modulated by NO, through both sGC-dependent and -independent mechanisms (Klyachko, Ahern, \& Jackson, 2001). In nerve terminals of the posterior pituitary, NO increases BK channel conductance, generating spike after hyperpolarization and also $\mathrm{Na}^{+}$ channels recovery from inactivation, so reducing action potential failures (Klyachko et al., 2001). These mechanisms may explain how NO modulates DA in NA.

However, IbTx known as a BK channel blocker, does not inhibit frequency-dependent effects of SIN-1 (NO donor) on DA release in NA. Several studies reported that NO-mediated DA release was independent of glutamate and GABA inputs (Hartung et al., 2011; Exley, Clements, Hartung, McIntosh, \& Cragg, 2008; Threlfell et al., 2010). NO also modulate the release of ACh in dorsal and ventral striatum (Trabace \& Kendrick, 2000). Notably, ACh on DA axons has an important role in controlling of DA release (Exley et al., 2008; Zhang \& Sulzer, 2004). Hartung et al. revealed that inhibition of nicotinic ACh receptors (nAChRs) in NA would prevent the effects of $\mathrm{NO}$ on DA transmission. It seems that NO has 2 different mechanisms to control DA release.

One mechanism is indirect, involving regulation of ACh input to switch nAChRs off. Shifting nAChRs off reduces DA release at low frequencies of stimulation, but increases sensitivity of DA release, and increased DA release at high frequencies can be due to $\mathrm{AAChR}$ desensitization (Zhang \& Sulzer, 2004).

The second mechanism of NO appears to be directly related to DA axons, and also increases evoked DA regardless of the stimulus frequency. Mechanistically, the rise in DA release by both direct and indirect actions of NO could be explained by a rise in vesicle fusion events as suggested for hippocampal synaptosomes by Meffert et al. (1996). NO may increase the docking and fusion of dopaminergic vesicles at dopaminergic terminals, leading to an increase in DA release independent of frequency of stimulations. In addition, NO may also increase the docking and fusion of cholinergic vesicles at cholinergic terminals, leading to an increase in ACh release that, as described earlier, could desensitize nAChRs with the effect of increasing DA release at high frequencies of stimulation.

There are different types of neurons that produce endogenous $\mathrm{NO}$ in NA such as nNOS-expressing interneuron network and GABAergic interneurons (French et al., 2005) Taken together, the exact role of nNOS-expressing neurons in NA is unknown; however, these neurons might have a role in promoting the transmission of DA in DA neurons. It is likely that NO has diverse target molecules, proposed effector mechanisms, and various reported outcomes on dopamine release.

\section{Role of Nitric Oxide in Dopamine Release in the Ventral Tegmental Area}

Opiate-induced reward is initiated in the ventral tegmental area (VTA). Opiate activates $\mu$-opioid receptors 
and increases the firing rate of dopamine cells in VTA. In addition, some studies revealed that NOS has been detected in VTA, and NO release from VTA neurons is involved in the control of DA release in the shell of the NA (Gholami, Zarrindast, Sahraei, \& Haeri-Rohani, 2003; Karami, Zarrindast, Sepehri, \& Sahraei, 2003; Sahraei et al., 2004a). Dopaminergic neurons of VTA respond strongly to stressful situations (Salehi, Eimani, Sahraei, \& Meftahi, 2015). Application of sodium nitroprusside (a NO generator) induces a dose-dependent increase in DA release in rat striatum slices and decreases DA uptake in synaptosomes of NA.

Recent studies showed that intra-VTA injection of Larginine (precursor of NO) does not induce conditioned place preference (CPP) by itself, but it increases the morphine response when injected with morphine during acquisition of CPP. The effects of intra-VTA application of L-NAME on the induction of CPP and acquisition of morphine-induced CPP by itself do not induce place preference, but co- application with morphine dose-dependently decreases morphine-induced place preference. Also, rewarding effect of cocaine, as cocaine self-application decreased by L-NAME and inhibition of NOS in the VTA, attenuates cocaine-induced sensitization in rats. Moreover, 7-nitronidazole (a selective neuronal NOS inhibitor) blocks CPP induced by cocaine, nicotine, and alcohol.

Repeated treatment with L-NAME may alter dopaminergic transmission and be associated with a decrease in the extracellular levels of DA in NA. It has been demonstrated that L-NAME by itself does not significantly alter the baseline concentrations of DA, but it may inhibit DA release much more in amphetamine-dependent rats. Additionally, L-arginine induces DA release from the striatum in vivo and L-NNA (an inhibitor of NO synthesis) markedly reduces the effect of L-arginine on DA release (Hosseini et al., 2016; Gholami et al., 2003; Karami et al., 2003; Sahraei et al., 2007).

Both L-arginine and L-NAME are able to acutely decrease the expression of morphine-induced CPP when given immediately before the test session. Studies showed that the reinforcing properties of ethanol and morphine are reduced by sodium nitroprusside (a generator of NO)

Thus, L-arginine may increase the firing rate of DA cells in VTA and DA release in NA, and consequently prevents the expression of CPP (Chalabi-Yani et al., 2015; Karami et al., 2003; Sahraei et al., 2007; Sahraei et al., 2004a). Dopamine in NA is an important modula- tor of the information transfer from the limbic to motor system. In addition, opioids increase dopaminergic turn over in NA, causing behavioral changes such as increased locomotion. For example, L-arginine administration increases morphine-induced locomotion (Gholami et al., 2002; Karami et al., 2003; Sahraei et al., 2004a). These findings support the role of NO in the actions induced by morphine.

\section{Role of Nitric Oxide on Nicotine-Induced Conditioned Place Preference}

Among many chemical compounds, nicotine is the most effective psychoactive material which confers tobacco's addictive properties (Chalabi-Yani et al., 2015). In the brain, neuronal $\alpha-7$ nAchRs with high $\mathrm{Ca}^{2+}$ permeability and high distribution in DA systems, are proposed to be the major targets of nicotine for reward induction (Sharma \& Vijayaraghavan, 2008; Gott \& Clementi, 2004; Gott, Zoli, \& Clementi, 2006; Wonnacott, Sidhpura, \& Balfour, 2005).

Several studies demonstrated substantial interaction of nicotine with DA and NO in the brain. NO has a high diffusion ability that can easily cross cell membranes (Schulman, 1997). Generation of NO in neurons depends on the elevation of $\mathrm{Ca}^{2+}$ concentration (Guix, Uribesalgo, Coma, \& Munoz, 2005). NOS is present in both origins and projections of mesolimbic and nigrostriatal neurons (French et al., 2005; Liu, Jin, Tang, \& Cui, 2008) and its co-existence with nicotinic receptors is another indicator of nicotine-NO interaction in these regions.

Nitric oxide can also inhibit monoamine transporters; so increases DA concentration in synapses. Nicotine can increase DA concentration in the striatum, NA, and other targets (Mifsud, Hernandez, \& Hoebel, 1989; Balfour, Wright, Benwell, \& Birrel, 2000). Moreover, NO can mediate burst firing of action potentials of dopaminergic neurons of the rat VTA induced by nicotine (Schilstorm et al., 2004). Furthermore, nicotine may indirectly increase NO concentration by stimulation of glutamatergic terminals and activation of high calcium permeability NMDA receptors on DA neurons in VTA (Garthwaite, Palmer, \& Moncada, 1989; Meftahi, Janahmadi, \& Eslamizade, 2014; Schilstorm et al., 2004).

Our laboratory experiments showed that nicotine-induced CPP was suppressed by nitrergic drugs, namely L-arginine and L-NAME. This suppression is mediated by the effect of these drugs on DA system. Consistent with previous studies, our investigation showed that nicotine induced a dose-dependent CPP in animals 
primed with nicotine and thus had prior exposure to the drug (Barzegari-Sorkheh, Oryan, \& Sahraei, 2012). It is highly likely that $\mathrm{nAChRs}$ located in different parts of the mesolimbic DA system, including VTA and NA (Clarke, Schwart, Pau, \& Pert, 1985) play a pivotal role in the reward effects of nicotine and induction of CPP.

\section{Role of Nitric Oxide on the Opioid-In- duced Locomotors Sensitization}

Some studies show that nitric oxide has a pivotal role in locomotion in drug-induced sensitized rats. The induction of locomotion after the application of a high dose of L-arginine has been observed. In addition, application of high dose of L-NAME produces a decline in locomotion. Inhibition of NOS can lead to decrease in locomotor activity by dopaminergic transmission. L-NAME has also been shown that has reduced analgesic effects and DA-mediated morphine effect on locomotion (Bahari et al., 2015; Sahraei et al., 2004a; Sahraei et al., 2004b; Zarrindast et al., 2003).

It has been demonstrated that the molsidomine (an NO donor) potentiated hyperactivity, which induced by amphetamine and cocaine and the locomotor hyperactivity evoked by amphetamine, cocaine, SKF 38393 (a dopamine D1 receptor agonist), and bromocriptine (a dopamine $\mathrm{D} 2$ receptor agonist) decreased by NOS inhibitors.

Some findings showed that behavioral sensitization to the locomotor-stimulating effects of apomorphine or morphine may be induced by a schedule of intermittent presentation. Previous studies showed an increase in postsynaptic DA receptor sensitivity after long-term administration of psychostimulant drugs. Local changes in the activity of DA pathways may contribute to sensitization (Gholami et al., 2003; Sahraei et al., 2004b; Zarrindast et al., 2003). Application of L-arginine increases morphine- and apomorphine-induced sensitization. Several studies showed that NOS activation increased DA and glutamate release.

These neurotransmitters are known to be essential for the induction of sensitization to psychostimulants. Therefore, it is possible that L-arginine, in combination with apomorphine or morphine, increases DA release in NA and so potentiates the induction of sensitization (Gholami et al., 2002; Sahraei et al., 2004a; Zarrindast et al., 2003). In contrast to the effect of L-arginine, NOS inhibition by L-NAME significantly and dose-dependently decreases the acquisition of both apomorphine- and morphine-induced sensitization (Zarrindast et al., 2003).

\section{Role of Nitric Oxide on Morphine-Induced Conditioned Place Preference in the Hippo- campus and Amygdala}

In addition to the mesolimbic DA system, other structures, including the hippocampus might play a role in reward system. Evidently, the dorsal hippocampus contains the highest concentration of DA. The hippocampus receives a dopaminergic projection originating predominantly in the VTA (Gholami et al., 2002; Karami, Zarrindast, Sepehri, \& Sahraei, 2002).

A lot of evidence suggests that NO may be involved in mediating the release of DA in the hippocampus. Further evidence indicated that NO plays a role in the modulation of dopaminergic effects elicited by morphine in the hippocampus. Moreover, the hippocampus may be involved in CPP (Karami et al., 2002; Zarrindast et al., 2003). It has been shown that long-term treatment with L-arginine attenuates morphine antinociception by increasing NOS activity and by decreasing the concentration of morphine in hippocampus.

However, application of L-arginine (NO precursor) into the hippocampal CA1 area during conditioning does not induce CPP, but increases the morphine-induced CPP without statistically significant interaction between the responses to morphine and L-arginine. These data suggest that the NO pathway in the CA1 area of the rat hippocampus could be involved in CPP (Karami et al., 2002; Meftahi et al., 2015; Sahraei et al., 2004b). It has been found that the application of NMDA receptor antagonist (MK-801) attenuates the development of opiate tolerance and dependence in rats. Evidence demonstrates an important role for DA in the hippocampus. Also, the dopaminergic system is involved in modulating the memory processes of the hippocampus.

Evidently, NO is a retrograde transmitter that signals presynaptic neurons, causing an increase in the release of dopamine. Therefore, NO is a neuronal messenger mediating the release of DA in the CA1 area of the rat hippocampus (Eslamizade et al., 2015; Ghodrat, Sahraei, Razjouyan, \& Meftahi, 2014; Gholami et al., 2003; Karami, et al., 2003; Karami et al., 2002). It has been proposed that damage to the dorsal hippocampus interferes with spatial learning. CPP is a learning paradigm, in that animals must remember the place and cues associated with drug administration, and recent studies indicated that the NO system might play a role in mediating this type of learning (Gholami et al., 2003; Karami et al., 2002). 
Furthermore, the central amygdala receives a wide range of ascending thalamus and brainstem inputs, including dopaminergic input from the ventral midbrain. It has been indicated that lesions of the amygdala impair performance in a CPP (Gholami et al., 2003; Sahraei et al., 2004a; Zarrindast et al, 2002). Intra-central amygdala administration of a single dose of L-arginine or L-NAME does not elicit any response regarding conditioning. LNAME has been shown to be ineffective as regards the morphine reinforcement properties. Furthermore, it has been shown that co-administration of L-arginine with morphine during conditioning, increases morphine-induced CPP and also induces an increase in locomotion.

These responses, which are induced by morphine plus Larginine, are suppressed by the administration of L-NAME (Gholami et al., 2003; Sahraei et al., 2004a; Zarrindast et al, 2002). Increase in NO level enhances the morphine conditioning (Zarrindast et al, 2002). This possibility can be supported by the data indicating that NO may play a role in morphine tolerance and dependence and also in the rewarding properties of opiates. Taken together, DA terminals in the amygdala are distributed primarily in the central nucleus of the amygdala. NO causes an increase in the release of DA. Therefore, NO may be a neuronal messenger mediating the release of DA in the central nucleus of the rat amygdala (Sahraei et al., 2004a-c; Zarrindast et al, 2002).

\section{Discussion}

Analysis of our research showed that NO may be an important neuronal messenger that mediated the release of DA in the different areas of the brain such as VTA, NA, hippocampus, and amygdala. The mechanism of NO action may be related to the changes in DA release in different rewarding areas of the brain. Several biases can be introduced by literature search and selection procedure. We might have missed unpublished trials, leading to publication bias. Therefore, further research is recommended investigating the mechanisms of NO action in opioid tolerance and dependency.

\section{Acknowledgments}

This work was supported by Neuroscience Research Center, Baqiyatallah University of Medical Sciences. The authors are thankful to Vice Chancellor of Research, Baqiyatallah University of Medical Sciences, for its financial support.

\section{Conflict of Interest}

All authors declared no conflict of interest.

\section{References}

Balfour, D. J., Wright, A. E., Benwell, M. E., \& Birrel, C. E. (2000) The putative role of extra-synaptic mesolimbic dopamine in the neurobiology of nicotine dependence. Behavioural Brain Research, 113(1), 73-83.

Barzegari-Sorkheh, A. A., Oryan, S., \& Sahraei, H. (2012). The role of nitric oxide in nicotine reward: a place preference study in rats. African Journal of Pharmacy and Pharmacology, 6(34), 2544-553.

Bahari, Z., Manaheji, H., Dargahi, L., Daniali, S., Norozian, M., Meftahi, G. H., et al. (2015). Time Profile of nNOS Expression in the Spinal Dorsal Horn after L5 Spinal Root Transection in Rats. Neurophysiology, 47(4), 287-94

Bahari, Z., Manaheji, H., Hosseinmardi, N., Meftahi, G. H., Sadeghi, M., Danialy, S., et al. (2014). Induction of spinal long-term synaptic potentiation is sensitive to inhibition of neuronal NOS in L5 spinal nerve transected rats. EXCLI Journal, 13, 751-60.

Chalabi-Yani, D., Sahraei, H., Meftahi, G. H., Hosseini, S. B., Sadeghi-Gharajehdaghi, S., Ali-Beig, H., et al. (2015). Effect of transient inactivation of ventral tegmental area on the expression and acquisition of nicotine-induced conditioned place preference in rats. Iranian Biomedical Journal, 19(4), 214-19.

Clarke, P. S., Schwart, R. D., Pau, S. M., Pert, C. B., \& Pert, A. (1985) Nicotinic binding in rat brain: autoradiographic comparison of 3H-acetylcholine, 3H-nicotine and 125I-a-bungarotoxin. Journal of Neuroscience, 5(5), 1307-315.

Cragg, S. J. (2006). Meaningful silences: how dopamine listens to the ACh pause. Trends in Neurosciences, 29(3), 125-31.

Di Chiara, G. (2002). Nucleus accumbens shell and core dopamine: differential role in behavior and addiction. Behavioural Brain Research, 137(1), 75-114.

Erfani, M., Sahraei, H., \& Meftahi, G. H. (2015). Study of the effects of Maternal Psychological and physical stress on Morphine-induced Tolerance in F2 NMRI Generation Mice. Advances in Bioresearch, 6(6), 134-40.

Eslamizade, M. J., Saffarzadeh, F. Mousavi, S. M. M., Meftahi, G. H., Hosseinmardi, N., Mehdizadeh, M., et al. (2015). Alterations in CA1 pyramidal neuronal intrinsic excitability mediated by $\mathrm{Ih}$ channel currents in a rat model of Amyloid beta pathology. Neuroscience, 305, 279-92.

Exley, R., Clements, M. A., Hartung, H., McIntosh, J. M., \& Cragg, S. J. (2008). Alpha6-containing nicotinic acetylcholine receptors dominate the nicotine control of dopamine neurotransmission in nucleus accumbens. Neuropsychopharmacology, 33(9), 2158-166.

French, S. J., Ritson, G. P., Hidaka, S., \& Totterdell, S. (2005). Nucleus accumbens nitric oxide immunoreactive interneurons receive nitric oxide and ventral subicular afferents in rats. Neuroscience, 135(1), 121-31 .

French, S. J., \& Totterdell, S. (2002). Hippocampal and prefrontal cortical inputs monosynaptically converge with individual projection neurons of the nucleus accumbens. Journal of Comparative Neurology, 446(2), 151-65.

Garthwaite, J., Garthwaite, G., Palmer, R. M., \& Moncada, S. (1989) NMDA receptor activation induces nitric oxide synthesis from arginine in rat brain slices. European Journal of Pharmacology: Molecular Pharmacology, 172(4), 413-16. 
Ghodrat, M., Sahraei, H., Razjouyan, J., \& Meftahi, G. H. (2014). Effects of a saffron alcoholic extract on visual short-term memory in humans: a psychophysical study. Neurophysiology, 4(3), 247-53.

Gholami, A., Haeri-Rohani, A., Sahraei, H., \& Zarrindast, M. R. (2002). Nitric oxide mediation of morphine-induced place preference in the nucleus accumbens of rat. European Journal of Pharmacology, 449(3), 269-77.

Gholami, A., Zarrindast, M. R., Sahraei, H., \& Haeri-Rohani, A. (2003). Nitric oxide within the ventral tegmental area is involved in mediating morphine reward. European Journal of Pharmacology, $458(1), 119-28$.

Gott, C. I., \& Clementi, F. (2004). Neuronal nicotinic receptors: from structure to pathology. Progress in Neurobiology, 74(6), 363-96.

Gott, C. I., Zoli, M., \& Clementi, F. (2006). Brain nicotinic acetylcholine receptors: native subtypes and their relevance. Trends in Pharmacological Sciences, 27(9), 482-91.

Groenewegen, H. J., \& Trimble, M. (2007). The ventral striatum as an interface between the limbic and motor systems. CNS Spectrums, 12(12), 887-92.

Guix, F. X., Uribesalgo, I., Coma, M., \& Munoz, F. J. (2005). The physiology and pathophysiology of nitric oxide in the brain. Progress in Neurobiology, 76(2), 126-52.

Hall, C. N., \& Garthwaite, J. (2009). What is the real physiological NO concentration in vivo? Nitric Oxide, 21(2), 92-103.

Hartung, H., Threlfell S., \& Cragg, S. J., (2011). Nitric oxide donors enhance the frequency dependence of dopamine release in nucleus accumbens. Neuropsychopharmacology, 36(9), 1811-822.

Hosseini, S. B., Sahraei, H., Mohammadi, A., Hatef, B., Meftahi, G. H., Chalabi-Yani, D., et al. (2015). Inactivation of the Nucl. Accumbens core exerts no effect on nicotine-induced conditioned place preference. Neurophysiology, 47(4), 295-301.

Hosseini, Y., Sahraei, H., Meftahi, G. H., Dargahian, M., Mohammadi, A., Hatef, B., et al. (2016). Analgesic and anti-inflammatory activities of hydro-alcoholic extract of Lavandula officinalis in mice: possible involvement of the cyclooxygenase type 1 and 2 enzymes. Revista Brasileira de Farmacognosia, 26(1), 102-08.

Ikemoto, S. (2007). Dopamine reward circuitry: two projection systems from the ventral midbrain to the nucleus accumbensolfactory tubercle complex. Brain Research Reviews, 56(1), 27-78.

Jeffery, D., Steketee, M., \& Kalivas, P. W. (2011). Drug wanting: behavioral sensitization and relapse to drug-seeking behavior. Pharmacological Reviews, 63(2), 348-65.

Karami, M., Zarrindast, M. R., Sepehri, H., \& Sahraei, H. (2002) Role of nitric oxide in the rat hippocampal CA1 area in morphineinduced conditioned place preference. European Journal of Pharmacology, 449(1), 113-19.

Karami, M., Zarrindast, M. R., Sepehri, H., \& Sahraei, H. (2003). Sulpiride injections into the medial septum reverse the influence of intra-medial septum injection of L-arginine on expression of place conditioning-induced by morphine in rats. Brain Research, 976(1), 30-40.

Kiss, J. P. (2000). Role of nitric oxide in the regulation of monoaminergic neurotransmission. Brain Reserch Bulletin, 52(6), 459-66.
Kiss, J. P., \& Vizi, E. S. (2001). Nitric oxide: a novel link between synaptic and no synaptic transmission. Trends Neuroscience, 24(4), 211-15

Klyachko, V. A., Ahern, G. P., \& Jackson, M. B. (2001). cGMP-mediated facilitation in nerve terminals by enhancement of the spike afterhyperpolarization. Neuron, 31(6), 1015-025.

Kraus, M. M., \& Prast, H. (2001). The nitric oxide system modulates the in vivo release of acetylcholine in the nucleus accumbens induced by stimulation of the hippocampal fornix/fimbriaprojection. European Journal of Neuroscience, 14(7), 1105-112.

Liu, H. Y, Jin, J., Tang, J. S., \& Cui, J. M. (2008). Chronic fatigue stress leads to up-regulation of nitric oxide synthase in the rat nucleus accumbens. Scandinavian Journal of Medicine \& Science in Sports, 18(6), 715-18.

Lonart, G., \& Johnson, K. M. (1994). Inhibitory effects of nitric oxide on the uptake of $[3 \mathrm{H}]$ dopamine and $[3 \mathrm{H}]$ glutamate by striatal synaptosomes. Journal Neurochemistry, 63(6), 2108-117.

Matsumoto, M., \& Hikosaka, O. (2009). Two types of dopamine neuron distinctly convey positive and negative motivational signals. Nature, 459(7248), 837-41.

Meffert, M. K., Calakos, N. C., Scheller, R. H., \& Schulman, H (1996). Nitric oxide modulates synaptic vesicle docking fusion reactions. Neuron, 16(6), 1229-236.

Meftahi, G., Ghotbedin, Z., Eslamizade, M. J., Hosseinmardi, N. \& Janahmadi, M. (2015). Suppressive effects of resveratrol treatment on the intrinsic evoked excitability of CA1 pyramidal neurons. Cell Journal (Yakhteh), 17(3), 532-39.

Meftahi, G. H., Janahmadi, M., \& Eslamizade, M. J. (2014). Effects of resveratrol on intrinsic neuronal properties of CA1 pyramidal neurons in rat hippocampal slices. Physiology and Pharmacology, 18(2), 144-55.

Mifsud, J. C., Hernandez, L., \& Hoebel, B. G. (1989). Nicotine infused into the nucleus accumbens increases synaptic dopamine as measured by in vivo microdialysis. Brain Research, 478(2), 365-67.

Morris, G., Schmidt, R., \& Bergman, H. (2010). Striatal actionlearning based on dopamine concentration. Experimental Brain Research, 200(3-4), 307-17.

Ohno, M., Arai, I., \& Watanabe, S. (1995). N-Methyl-D-aspartate stimulates dopamine release through nitric oxide formation in the rat nucleus accumbens of rats. Brain Research, 699(2), 332-35.

Pogun, S., \& Michael, A. (1994). Nitric oxide inhibits [3H] dopamine uptake. Brain Research, 641(1), 83-91.

Prast, H., \& Philippu, A. (2001). Nitric oxide as modulator of neuronal function. Progress in Neurobiology, 64(1), 51-68.

Prast, H., Tran, M. H., Fischer, H., \& Philippu, A. (1998). Nitric oxideinduced release of acetylcholine in the nucleus accumbens: role of cyclic GMP, glutamate, and GABA. Journal of Neurochemistry, 71(1), 266-73.

Robinson, T. E., \& Berridge, K. C. (2003). Addiction. Annual Review Psychology, 54, 25-53.

Rodrigo, J., Springall, D. R., Uthenthal, O., Bentura, M. L., AbadiaMolina, F., Riveros-Moreno, V., et al. (1994). Localization of nitric oxide synthase in the adult rat brain. Philosophical Transactions of the Royal Society, 345(1312), 175-221. 
Sahraei, H., Falahi, M., Zarrindast, M. R., Sabetkasaei, M., Ghoshooni, H., \& Khalili, M., (2004a). The effect of nitric oxide on the acquisition and expression of nicotine-induced conditioned place preference in mice. European Journal of Pharmacology, 503(1-3), 81 87.

Sahraei, H., Pirzadeh-Jahromi, G., Noorbakhshnia, M., Asgari, A., Haeri-Rohani, A., Khoshbaten, A., et al, (2004b). Involvement of nucleus accumbens in L-arginine- induced conditioned place preference in rats. Journal of Behavioral Pharmacology, 15(7), 473-80.

Sahraei, H., Poorheidari, G. M., Foadaddini, M., Khoshbaten, A., Asgari, A., Noroozzadeh, A., et al. (2004c). Effect of nitric oxide on morphine self-administration in rat. Journal of Pharmacology. Biochemistry and Behavior, 77(1), 111-16.

Sahraei, H., Zarei, F., Eidi, A., Oryan, S., Shams, J., Khoshbaten A., et al. (2007). The role of nitric oxide within the nucleus accumbens on the acquisition and expression of morphine-induced place preference in morphine sensitized rats. European Journal of Pharmacology, 556 (1), 99-106.

Salehi, M., Eimani, H., Sahraei, H., \& Meftahi, G. H. (2015). Stress can changes reward system function in second-generation (F2): a review. Advances in Bioresearch, 6(5), 4-14

Schilstorm, B., Mameli-Engvall, M., Rawall, N., Grillner, P., Jardemark, K., \& Svensson, T. H. (2004). Nitric oxide is involved in nicotine-induced burst firing of rat ventral tegmental areadopamine neurons. Neuroscience, 125(4), 957-64.

Schulman, H. S. (1997). Nitric oxide: a spatial second messenger. Molecular Psychiatry, 2(4), 296-99.

Schultz, W. (2002). Getting formal with dopamine and reward. Neuron, 36(2), 241-63.

Schultz, W. (2010). Dopamine signals for reward value and risk: basic and recent data. Behavioral Brain Function, 6, 24. doi 10.1186/1744-9081-6-24

Sesack, S. R., \& Grace, A. A. (2010). Cortico-basal ganglia reward network: microcircuitry. Neuropsychopharmacology, 35(1), 27-47.

Sharma, G., \& Vijayaraghavan, S. (2008). Nicotinic receptors: role in addiction and other disorders of the brain. Substance Abuse: Research and Treatment, 1, 81-95.

Siggins, G. R., Martin, G., Roberto, M., Nie, Z., Madamba, S., \& DeLecea, L. (2003). Glutamatergic transmission in opiate and alcohol dependence. Annals of the New York Academy of Sciences, 1003(1), 196-211.

Stewart, T. L., Michel, A. D., Black, M. D., \& Humphrey, P. P. (1996) Evidence that nitric oxide causes calcium-independent release of $[3 \mathrm{H}]$ dopamine from rat striatum in vitro. Journal Neurochemistry, $66(1), 131-37$.

Threlfell, S., Clements, M. A., Khodai, T., Pienaar, I. S., Exley., R., Wess, J., et al., (2010). Striatal muscarinic receptors promote activity dependence of dopamine transmission via distinct receptor subtypes on cholinergic interneurons in ventral vs dorsal striatum. Journal of Neuroscience, 30(9), 3398-408.

Trabace, L., Cassano, T., Tucci, P., Steardo, L., Kendrick, K. M., \& Cuomo, V. (2004). The effects of nitric oxide on striatal serotoninergic transmission involve multiple targets: an in vivo microdialysis study in the a wake rat. Brain Research, 1008(2), 293-8.

Trabace, L., \& Kendrick, K. M. (2000). Nitric oxide can differentially modulate striatal neurotransmitter concentrations via soluble guanylate cyclase and peroxynitrite formation. Journal of Neurochemistry, 75(4), 1664-674.

Wiesinger, H. (2001). Arginine metabolism and the synthesis of nitric oxide in the nervous system. Progress in Neurobiology, 64(4), 365-91.

Wonnacott, S., Sidhpura, N., \& Balfour, D. J. (2005). Nicotine: from molecular mechanisms to behavior. Current Opinion Pharmacology, 5(1), 53-59.

Yamada, K. (2008). Endogenous modulators for drug dependence. Journal of Biological and Pharmaceutical Bulletin, 31(9), 1635-638.

Yaribeygi, H., Sahraei, H., Mohammadi, A. R., \& Meftahi, G. H. (2014). Saffron (Crocus sativus L.) and morphine dependence: A systematic review article. American Journal of Biology and Life Sciences, 2(2), 41-45.

Zahm, D. S. (2000). An integrative neuroanatomical perspective on some subcortical substrates of adaptive responding with emphasis on the nucleus accumbens. Neuroscience Biobehavioral Review, 24(1), 85-105.

Zarrindast, M. R., Gholami, A., Sahraei, H., \& Haeri-Rohani, A. (2003). Role of nitric oxide in the acquisition and expression of apomorphine- or morphine- induced locomotor sensitization. European Journal of Pharmacology, 482(1-3), 205-13.

Zarrindast, M. R., Karami, A., Sepehri, H., \& Sahraei, H. (2002). Influence of nitric oxide on morphine-induced conditioned place preference in the rat central amygdale. European Journal of Pharmacology, 453(1), 81-89.

Zhang, H., \& Sulzer, D. (2004). Frequency-dependent modulation of dopamine release by nicotine. Nature Neuroscience, 7(6), 581-2. 